\title{
Statyba
}

\section{INNOVATIVE DIVISION OF INFORMATION SYSTEMS OF BUILDING ENTERPRISES ACCORDING TO THE METHODS OF ECONOMY AND PROCESS TECHNOLOGY}

\section{S. Reader}

To cite this article: S. Reader (1997) INNOVATIVE DIVISION OF INFORMATION SYSTEMS OF BUILDING ENTERPRISES ACCORDING TO THE METHODS OF ECONOMY AND PROCESS TECHNOLOGY, Statyba, 3:12, 82-89, DOI: 10.1080/13921525.1997.10531371

To link to this article: https://doi.org/10.1080/13921525.1997.10531371

曲 Published online: 26 Jul 2012.

Submit your article to this journal $2 \pi$

山ll Article views: 39 


\section{KONZEPTION ZUR NEUGLIEDERUNG DER BAUBETRIEBLICHEN INFORMATIONSSYSTEME NACH BETRIEBSWIRTSCHAFTLICHEN UND VERFAHRENSTECHNISCHEN METHODEN}

\section{S. Raeder}

\section{Voranstellung}

Die Abwicklung von Bauprojekten ist heute ohne Einsatz der Informationstechnik nicht mehr denkbar. Das betrifft sowohl den Überwachungsbereich, der dem Auftraggeber zuzuordnen ist, als auch den operativen, der durch den Auftragnehmer wahrgenommen wird. Die Leistungsbilder in diesen beiden Bereichen unterscheiden sich grundlegend voneinander.

Die Gliederung der Projektabwicklung in die vier Teilvorgänge [1]

- Planung und Konstruktion,

- Genehmigungsphase,

- Bauvorbereitung und

- Bauausführung

zeigt deutlich, daß der operative Bereich erst mit der Auftragserteilung und mit der erteilten Baugenehmigung beginnt. Die davor liegenden Teilvorgänge Planung und Konstruktion sowie Genehmigungsphase und Bauvorbereitung können also nicht dem unmittelbaren Baugeschehen zugerechnet werden, da sie zwar notwendige, aber periphere Leistungen enthalten. Dies wird schon durch die Annahme des Bieterangebotes bei Vertragsabschluß durch den Auftraggeber dokumentiert. Hier wird der Auftragnehmer vertraglich zur vereinbarten Leistungserbringung verpflichtet und der Auftraggeber zur Zahlung der vereinbarten Vergütung. Damit ist die Aufgabenverteilung bei der Projektabwicklung eindeutig geregelt. Auf diese Weise entstehen für die Projektabwicklung unterschiedliche Informationssysteme.

In der Systemgestaltung sind diese Wesensunterschiede strukturbestimmend. Zur Erfüllung der Ziele ist bei der Organisation der Informationssysteme auf die Teilfunktionen z. B. der Bauüberwachung bzw. des operativen Bereiches zu achten. In der weiteren Betrachtung sind die Gedanken der Konzeption dem operativen Bereich zugewandt.

\section{Die Notwendigkeit neuer baubetrieblicher Informationssysteme}

Die klassische Gliederung eines Bauunternehmens umfaßt grob zwei Hauptbereiche, einen technischen und einen kaufmännischen Bereich. Dieser hierarchischen Struktur entsprechend sind die Ingenieuraufgaben und ebenso die Aufgaben der Betriebswirtschaft streng voneinander getrennt. Das führt zu Überschneidungen im Kompetenzbereich auf den Führungsebenen und so zu Informationsverzögerungen bis hin zu partiellen Informationsverlusten. Diese Nachteile sind weder durch Aufklärung der Personalebenen noch durch Anordnungen $\mathrm{zu}$ beseitigen. Sie wirken sich unmittelbar als wirtschaftliches Defizit aus. Zur Vermeidung dieser Unzulänglichkeiten sind Ziele zu definieren, die einen Handlungsrahmen schaffen, in dem Möglichkeiten zur Verknüpfung der Kompetenzen zwischen den verschiedenen Ebenen zwangsläufig gegeben sind.

Die Entscheidungen und vor allen Dingen Grundsatzentscheidungen liegen überwiegend auf höheren Managementebenen. Solche Entscheidungsprozesse werden deshalb unterschiedliche Folgewirkungen auf die mittleren Managementebenen ausüben. Ein gut funktionierendes Informationssystem hat diese Auswirkungen zu wichten.

Wenn auch ein Informationssystem im wesentlichen darauf ausgerichtet ist, die Informationen und die Entscheidungsprozesse zu verbessern, so liegt ihm doch das Primärziel der Entscheidungsoptimierung zugrunde. Das führt dazu, daß gute Informationen gesammelt werden müssen, um zu besseren Entscheidungen zu gelangen.

Für Bauunternehmen bedeutet dies, da die Bauproduktion fast ausschließlich auf Baustellen stattfindet, daß es sich um baubetriebliche Informationssysteme handelt. Der Organisationsbereich ist die Baustelle und die Datenerhebung findet auch auf dieser statt. Das Informationssystem erhält also, um seiner Dokumentationsfunktion 
gerecht zu werden, das Datenmaterial von der Baustelle. Mit diesen Daten und der Steuerungsfunktion des Informationssytems lassen sich dann durch das Planungsmodell Dispositionen optimieren, so daß bessere Entscheidungen ermöglicht werden. Systeme dieser Art für baubetriebliche Prozesse stehen zur Zeit nur bedingt zur Verfügung. Gäbe es sie, so würden sie eine bessere Transparenz der Bauproduktion bewirken.

Die bestehenden Baustellen-Informationssysteme, wie z. B. nach Wirth und Cron [2] in Deutschland, decken zwar mit den aufgestellten Controllingthesen den Teil der Informationsbeschaffung durch den SOLL-IST-Vergleich ab, werden aber der Umsetzung der Informationen in Aktionen nicht ausreichend gerecht. Der SOLL-IST-Vergleich auf allen Ebenen der Baustellen in vielen Stufen liefert eine immense Zahl von Informationen, die in der Form nicht zur Selektion von optimalen und alternativ möglichen Aktionen führen können.

Das Konzept eines Beratersystems für Baustellenleiter nach Gehri ([2] S. 91 ff.), das als Jobmodell bezeichnet ist, zeigt einen anderen Weg auf. Hier stellt der Job eine klar definierte und mit dem Leistungsverzeichnis verknüpfte Ablaufeinheit dar. Jeder Job kann also mehrere Leistungspositionen enthalten. Es wird damit eine geringere Anzahl an Jobpositionen als an Leistungspositionen geben, was zu Entscheidungserleichterungen führen kann. Durch die Verknüpfungen lassen sich aber keine direkten Aussagen mehr über Abweichungen von den vertraglich vereinbarten Leistungspositionen machen. Das aber bedeutet einen Nachteil in der Handhabung bei der Bauabwicklung.

Insgesamt zeigt es sich, daß die BaustellenInformationssysteme eine Vielzahl an Informationen aus dem Produktionsprozeß für die Entscheidungsprozesse der Einheit Baustelle anbieten. Es gibt also sehr viele Einzelentscheidungen, die wiederum für eine Gesamtbetrachtung von Nachteil sind.

Ein gegenwartsbezogenes und allgemeingültiges baubetriebliches Informationssystem darf diese Nachteile nicht aufweisen. Die Managementebene muß die Managementfunktionen Kommunikation und Entscheidung durchsetzen. Die Phasen Planen, Realisieren und Kontrollieren bilden dabei den Grundzyklus. Eine Wichtung der Funktionen ist von der Managementebene vorzunehmen. Beim baubetrieblichen Geschehen überwiegt der Überwachungsanteil. Dementsprechend groß fällt auch der
Steuerungsanteil aus. Beide Anteile müssen zu Gunsten der Transparenz der Produktionsabläufe möglichst gering gehalten werden. Diese Forderungen führen zu aggregierten Entscheidungsprozessen. Das Informationssystem hat dies im Aufbau der Dokumentations- und Steuerungsfunktion zu berücksichtigen.

\section{Ganzheitliche Transparenz durch neues baubetriebliches Informationssystem}

Die angesprochenen Baustellen-Informationssysteme gehen von Kostenbetrachtungen aus. Dabei werden die SOLL-Werte auf der Kalkulation, die der Strukturierung des Leistungsverzeichnisses entspricht, aufgebaut. Gehri weicht davon ab und wählt den Job. Bei allen bedeutet das ein abschnittsweises Vorgehen in einer Vielzahl von Schritten. Des weiteren werden in allen Fällen Kostenartenbetrachtungen vorgenommen. Ebenso ist die besondere Beachtung des Elementes der Lohnkosten allen Systemen gemein. Für die Durchsetzung der sequentiellen Funktionen Planen, Realisieren und Kontrollieren ergibt sich damit ein ungeheurer Aufwand, der sich auch nicht durch die Informationstechnik wesentlich erleichtern läßt. Der Aufwand ergibt sich aus der Datenerhebung und der Wertung der einzelnen Vorgänge. Durch die Kostenartenbetrachtungen aller Vorgänge der Einzelkosten der Teilleistungen ergibt sich zwar ein umfangreiches vielschichtiges Bild, das sich jedoch in dieser Form für Vergleiche im regelmäßigen Zyklus $\mathrm{zu}$ umfangreich und aufwendig erweist.

Neben diesen Kostenbetrachtungen sind die Pflege der Termine und die vergleichenden Beobachtungen der eingesetzten Ressourcen sowie die Dokumentation der qualitätssichernden Maßnahmen notwendig. Hier zeigt sich insgesamt eine Anhäufung von unproduktiven Tätigkeiten, die in dieser Form zunächst nur kostenverursachend wirken und wenig effizient erscheinen. Die Akzeptanz zur Anwendung solcher die Effizienz der Bauabwicklung verbessernden Maßnahmen bleibt deshalb dennoch gering.

Die traditionellen Verfahren der Baukalkulation zur Ermittlung der Einheitspreise und der Angebotssumme gehen nicht auf die Produktionsfunktionen der Betriebswirtschaftslehre zurück [3], sondern sind besonders dafür entwickelte Kostenfunktionen. Die Produktions- und Kostenfunktionen der Betriebswirtschaftslehre gelten hier als weniger geeignet. Für die Kostenfunktion wurde die Form 


$$
K=f_{1}(Q)+\sum\left\{f_{2}\left(Q_{j}\right)\right\}+f_{3}(Z)+K_{42}+C
$$

mit $K=$ Gesamtkosten der Baustelle

$Q=$ Gesamtpotential der Baustelle

$Q_{j}=$ Potential des Prozesses $(j)$

$Z=$ Bauzeit

$K_{42}=$ Leerkosten der Fertigungsstraßen

$C=$ konstante Kosten im Sinne der Ablaufplanung

entwickelt ([3], S. 578). In dieser Funktion sind alle Kostenanteile der Produktionseinheit Baustelle enthalten. In der Bauauftragsrechnung hat sich die Anwendung dieser Funktionsform bewährt. Zur Beurteilung der Effektivität des Produktionsablaufes ist die Gesamtkostendarstellung in dieser Weise ungeeignet. Ausreichend wäre die Betrachtung solcher Kostenanteile, die von Leistungen hervorgerufen werden, die vorherrschend den operativen Bereich bestimmen. Dies bietet den Vorteil, daß eine Funktion dieser Art besser für Steuerungszwecke geeignet ist.

Diese Aspekte zwingen dazu, bei der Entwicklung eines neuen baubetrieblichen Informations- systems Prioritäten zu setzen. Wichtig ist dabei, daß Klarheit besteht, ob Kommunikations-, Steuerungsoder Entscheidungsprozesse Prioritäten haben sollen. Entsprechend sind die kybernetischen Elemente auszuwählen, um repräsentative Steuerungsgrößen zu erhalten oder geeignete Informationen für eine optimale Entscheidung.

Aus der Strukturierung der Bauunternehmen (Dritter Katalog) ergeben sich die unternehmerischen Bauaufgaben (Abbildung 1). Überwiegend sind das die operativen Aufgaben des Auftragnehmers. Von Bedeutung sind dabei die ingenieurmäßigen und die betriebswirtschaftlichen Rahmenbedingungen für die Bauproduktion und Prozeßsteuerung.

Die ingenieurmäßigen Rahmenbedingungen basieren auf den Normen und sind durch die wissenschaftlich begründeten baubetrieblichen Erkenntnisse abgesichert. Die technologischen Zusammenhänge der Produktionsprozesse und der Logistik sind auf dieser Grundlage disponierbar. Gestützt wird diese Planung auf den Ausschreibungsunterlagen des Auftraggebers (Erster Katalog) und dem Angebot des Auftragnehmers

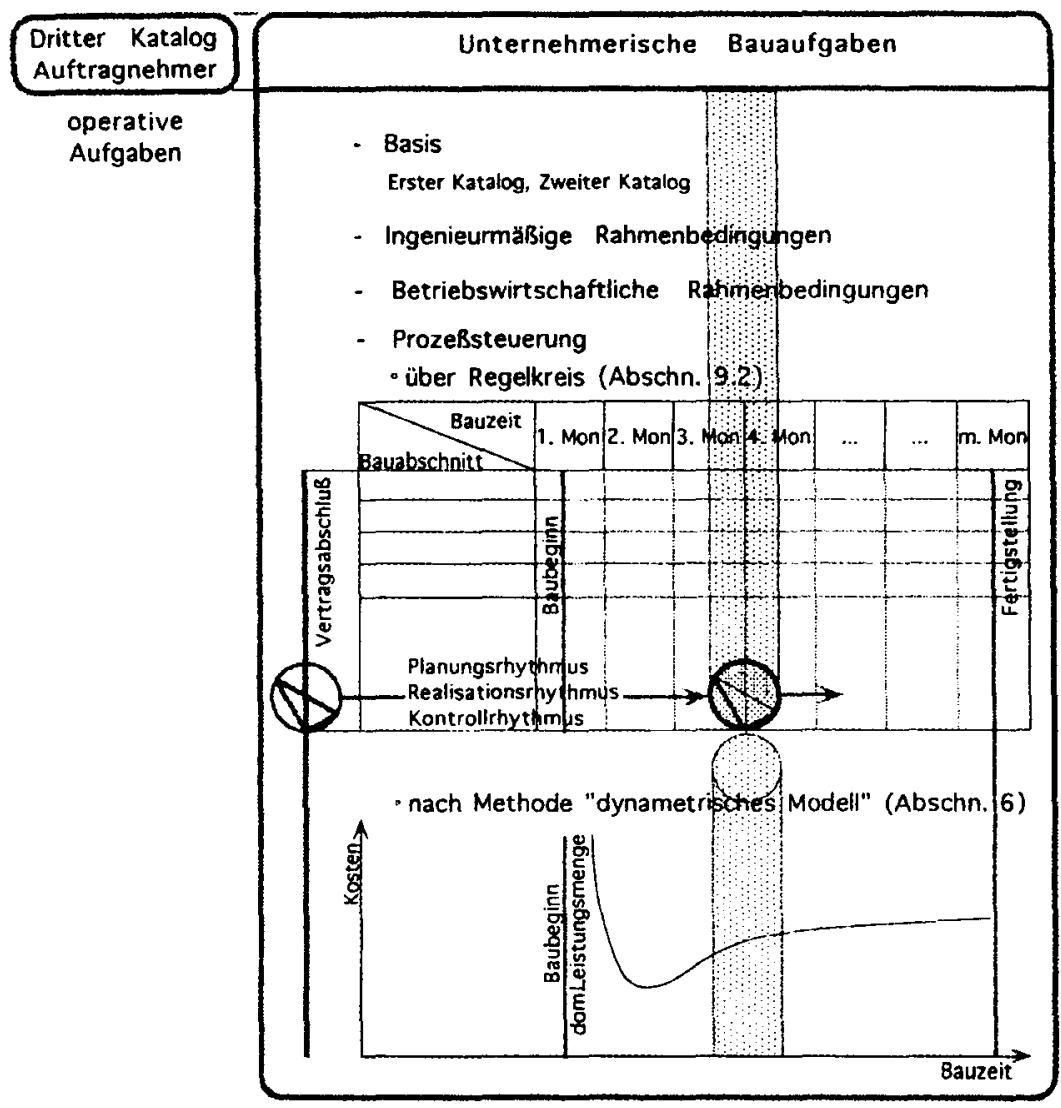

Abb. 1. Dritter Katalog - unternehmerische Bauaufgaben 
(Zweiter Katalog). Gemeinsam bilden sie den Kanon des logisch organisierten Baubetriebes. Dadurch ist die vertragsgemäße Leistungserbringung und die Entrichtung der vereinbarten Vergütung geregelt.

Bei der zeitlichen Gestaltung des Produktionsgeschehens ist ein prozeßorientierter Ablaufplan zu entwickeln, der alle Verknüpfungen erfaßt und einen wirtschaftlichen Ablauf auf der Grundlage des Rahmenterminplanes des Auftraggebers ermöglicht. Die zum Einsatz vorgesehenen Bauverfahren sind bereits in dieser Phase durch Verfahrensvergleiche $\mathrm{zu}$ bestimmen. Durch die operative Planung sollten

- die Angebotskalkulation als Arbeitskalkulation erstellt,

- die Mengen dem Erkenntnisstand angepaßt,

- die vorgesehenen Bauverfahren berücksichtigt,

- das gewerbliche Personal festgelegt,

- die Vorgangsdauern präzisiert,

- die Ablaufplanung verfeinert,

- die Gemeinkosten der Baustelle bestimmt und

- die Verfügbarkeit der Ressourcen durch die Logistik gesichert

werden

Die betriebswirtschaftlichen Rahmenbedingungen ergeben sich aus dem Bereich der Baubetriebsrechnung, die zur organisatorischen Einheit der Kosten- und Leistungsrechnung gehört. Hier werden alle durch die Leistungserbringung entstandenen Umsatzströme mengen- und wertmäßig erfaßt. Zur Kosten- und Leistungsrechnung zählt auch die Bauauftragsrechnung. Beide Bereiche, die Baubetriebsrechnung als auch die Bauauftragsrechnung verfolgen trotz unterschiedlicher Aufgaben das gemeinsame Ziel der Erfolgsorientierung. Eine wesentliche Aufgabe der Baubetriebsrechnung besteht in der Systematisierung, Zuordnung und Sammlung der unterschiedlichen Kosten entsprechend dem Kalkulationsschema zu Kostenstellen, Kostenarten, Kostenträgern, Gemeinkosten und Allgemeinen Geschäftskosten, so daß eine Weiterverwendung für Informationszwecke jederzeit möglich ist. Der Informationswert hängt davon $a b$, welchen Stellenwert die Beteiligten diesem Verfahren beimessen.

Die Prozeßsteuerung im operativen Bereich hat sich an den gesetzten Zielen der operativen Planung $\mathrm{zu}$ orientieren und bei Regelabweichungen Kompensationen vorzunehmen. Die ingenieurmäßigen und betriebswirtschaftlichen Belange der jeweiligen
Fachdisziplinen sind dabei zu berücksichtigen. Auf vertraglicher Basis sind die

- Leistungen,

- Kosten,

- Ressourcen und

- Bauprozesse

zu planen. Die Steuerungsparameter, die für den Kontrollprozeß die IST- und SOLL-Werte liefern, sind zu entwickeln und einzuführen. Sie müssen, um ganzheitliche Transparenz zu gewähren, aus allen drei Katalogen des Kanons des logisch-organisierten Baubetriebes [4] hervorgehen.

\section{Struktur des neuen baubetrieblichen Informationssystems}

Aus der Abwägung der Priorität nach dem Zweck des Systems bestimmen sich die Steuerungsparameter. Ein in sich logisch organisierter Baubetrieb regelt sich von selbst. Daraus ergibt sich, daß es zunächst keiner Steuerung bedarf. Steuerungsfunktionen sind erst dann wahrzunehmen, wenn Einflüsse von außen Störungen hervorrufen. Die Informationen über die Abweichungen von Führungsgrößen (SOLL-Werte) zwingen zu Aktionen der Kompensation. Dies sind die Merkmale von Entscheidungsprozessen. Damit müssen über die Informationssysteme Steuerungs- und Entscheidungsmöglichkeiten gegeben sein.

Aus den äußerst komplexen Zusammenhängen der Produktionsprozesse der Baustellenfertigung sind die variablen Führungsgrößen dem Gesamtsystem anzupassen, das heißt, es soll nicht mit Teilsystemen gearbeitet werden, denn das widerspricht der Konzeption der ganzheitlichen Betrachtung. Das Informationssystem erhält so eine gewisse Trägheit und bleibt dadurch in seiner Aussagefähigkeit stabil. Für den Systemaufbau eignet sich die Kostentheorie der Betriebswirtschaftslehre. Die Systemelemente sind aus dem betriebswirtschaftlichen als auch aus dem technologischen Bereich auszuwählen und durch ihre gegenseitigen Beziehungen zu strukturieren. Auf diese Weise lassen sich die kausalen Relationen der Bauverfahren, Bauzeit, Baukosten und Qualität erfassen.

Die allgemeine Kostenfunktion lautet

$$
K=f(m)
$$

beziehungsweise

$$
K=K_{f}+K_{v}(m)
$$




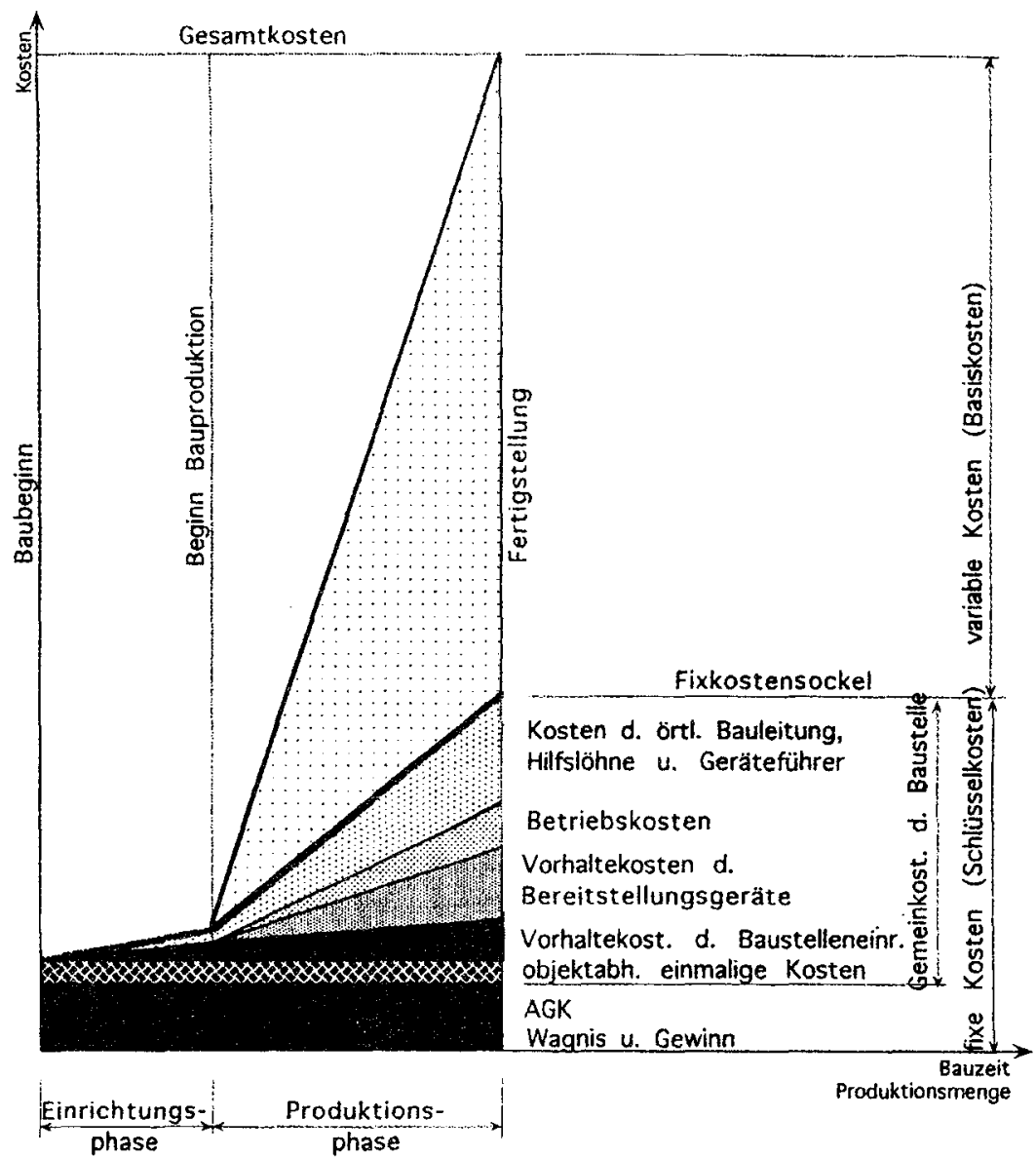

Abb. 2. Schematische Darstellung der Kostenstruktur eines Bauprojektes

mit K Gesamtkosten

$K_{f}$ fixe Kosten

$K_{v}(m)$ variable Kosten.

In dieser Kostenfunktion sind die fixen und variablen Kosten jeweils für sich ausgewiesen. Dabei enthalten die fixen Kosten alle zeitabhängigen und die variablen Kosten alle mengenabhängigen Kostenanteile. Das bedeutet, daß sich die Einzelkosten der Teilleistungen mit den dazugehörenden Mengen in den variablen Kosten widerspiegeln und die Gemeinkosten der Baustelle sowie die Allgemeinen Geschäftskosten und die Wagnisse im Fixkostenblock enthalten sind (Abbildung 2). Bei Anwendung der Vollkostenrechnung lassen sich so möglichst viele Kosten verursachungsgerecht dem Kostenträger zuweisen.

Nach dieser Zuordnung sind für das Gesamtsystem alle kybernetischen Elemente, die Führungsgrößen sein sollen, zu bestimmen. Die Anzahl dieser Elemente ist wegen der Praktikabilität möglichst gering zu halten. Die Elemente müssen so gewählt werden, daß alle Produktionsfaktoren erfaßt sind.
Der mit Preisen bewertete Verbrauch der Produktionsfaktoren, der durch die Leistungserbringung verursacht wird, stellt die Kosten dar. So wird in der Gesamtkostenfunktion das ganze vertraglich geforderte Leistungsspektrum erfaßt. Damit läßt sich mit der Kosten- und Leistungsbetrachtung das Informationssystem aufbauen.

Die Kosten sind in streng betriebswirtschaftlichem Sinne eine Funktion der Ausbringungsmenge m. Im baubetrieblichen ProduktionsprozeB existiert eine solche Menge nicht. Zur Anwendung der Kostenfunktion muß eine adäquate Menge definiert werden. Im Produktionsprozeß fallen unterschiedliche Mengen in mehreren Leistungspositionen gleichzeitig an, das heißt, daß aus dieser Vielzahl eine Leistungsmenge ausgewählt werden muß, die das Baugeschehen prägt und möglichst über die gesamte Bauzeit erbracht wird. Das wird je nach Bauprojekt eine andere Leistungsmenge sein. Sie läßt sich als dominierende Leistungsmenge $m^{o}$ bezeichnen und stellt eine der Ausbringungsmenge adäquate Größe in der Gesamtkostenfunktion dar.

Aus der Analyse der variablen Kosten 


$$
K_{v}=k_{v} \cdot m
$$

mit den variablen Einheitskosten

$$
k_{v}=k_{v_{L o}}+k_{v_{M a t}}+k_{v_{\text {Gera }}}+k_{v_{F L}}+\ldots
$$

wobei $k_{v_{L o}}$ Einheitskostenanteil aus Lohn

$k_{v_{\text {Mat }}}$ Einheitskostenanteil aus Material

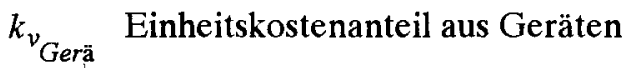

$k_{v_{F L}} \quad$ Einheitskostenanteil aus

Fremdleistungen

ergeben sich weitere kybernetische Elemente. Die Einheitskostenanteile des Lohnes setzen sich aus der Arbeitszeit AT $[\mathrm{h}]$ und dem Mittellohn ML $\left[\frac{D M}{h}\right]$, bezogen auf die dominierende Leistungsmenge $\mathrm{m}^{\circ}$ [ME], zusammen. Die übrigen Einheitskostenanteile können als Einheitsstoffkosten aller Restkosten $k_{v_{S t}}\left[\frac{D M}{M E}\right]$ bezogen auf die Mengeneinheit der dominierenden Leistungsmenge zusammengefaßt werden. Mit diesen Festlegungen lassen sich die variablen Einheitskosten als

$$
k_{v}=\frac{A T \cdot M L}{m^{o}}+k_{v_{S t}}
$$

darstellen.

Die allgemeine Kostenfunktion erhält unter Berücksichtigung obiger Einführungen und Definitionen die Form [4]

$$
K=K_{f}+\frac{A T}{m^{o}} \cdot M L\left\{1+\frac{k_{v_{S t}}}{M L} \cdot \frac{m^{o}}{A T}\right\} \cdot m
$$

mit $m$ als Ausbringungsmenge;

sie entspricht der dominierenden

Leistungsmenge.

Eine analytische Betrachtung zeigt, daß der Quotient $\frac{A T}{m^{\circ}}$ einen den Aufwandswerten ähnlichen Ausdruck der Bauauftragsrechnung darstellt. Er kann als fiktiver Aufwandswert angesehen werden. Infolgedessen soll er als Einheit behandelt werden. Um das zu gewährleisten, wird der Terminus $D Y C H A=\frac{A T}{m^{\circ}}\left[\frac{h}{M E}\right]$ (dynamerische Charakteristik) eingeführt. Die „dynametrische Charakteristik“ ist Hauptelement der Kostengleichung und drückt den Anteil der Verfahrenstechnik aus.
Als weiterer bemerkenswerter dimensionsloser Faktor gilt der Klammerausdruck. Er wird mit dem Buchstaben $\varepsilon$ bezeichnet und beinhaltet die Einheitsstoffkosten der Einzelkosten der Teilleistungen in abgeminderter Form. In ihm finden sich die betriebswirtschaftlichen Kostenanteile wieder.

Die Schreibweise der allgemeinen Kostenfunktion unter Berücksichtigung betriebswirtschaftlicher und verfahrenstechnischer Methoden ergibt sich damit zu

$$
K=K_{f}+D Y C H A \cdot M L \cdot e \cdot m .
$$

Die Darstellung des Gesamtkostenverlaufes der Kostenfunktion (Abbildung 3) im zeitlichen Ablauf der Bauprozesse gliedert den Baubetrieb in verschiedene Produktionsabschnitte, in denen unterschiedliche Kostenträger wirken. Die kybernetischen Elemente, welche Führungsgrößen darstellen, enthält die Gesamtkostenfunktion. Insgesamt sind es fünf Elemente, nämlich

- der Fixkostenblock $\left(K_{f}\right)$,

- der Mittellohn ( $M L)$,

- die Arbeitszeit $(A T)$ in der DYCHA,

- die dominierende Leistungsmenge $\left(m^{\circ}\right)$ in der $D Y C H A$ und

- die Einheitskosten der Stoffe $k_{v_{S t}}$ im Faktor $\varepsilon$.

Mit diesen prozeßorientierten kybernetischen Elementen wird das baubetriebliche Informationssystem den betriebswirtschaftlichen und verfahrenstechnischen Forderungen gerecht.

\section{Zusammenfassende Schlußbetrachtung}

Baubetriebliche Informationssysteme sind dynamische offene Systeme. Sie besitzen aktive Elemente, die über materielle Versorgungsgrößen gekoppelt sind. Ebenso beinhalten sie Elemente, die Beziehungen zur Umgebung aufweisen, das sind z. B. die Randelemente der Ressourcenbeschaffung sowie die Beziehungen zum Auftraggeber. Beim Aufbau des Systems ist davon auszugehen, daß die Ausrichtung auf den Bauauftrag mit seinen vertraglichen Vereinbarungen zu erfolgen hat mit der Notwendigkeit als Ziel, einen Gewinn zu erwirtschaften. Alle Abweichungen von den Führungsgrößen sind deshalb durch Rückkoppelung und Kompensation zu beseitigen. Diese regulierenden Eigenschaften besitzt das entwickelte baubetriebliche Informationssystem. Damit ist es als kybernetisches System zu bezeichnen. 


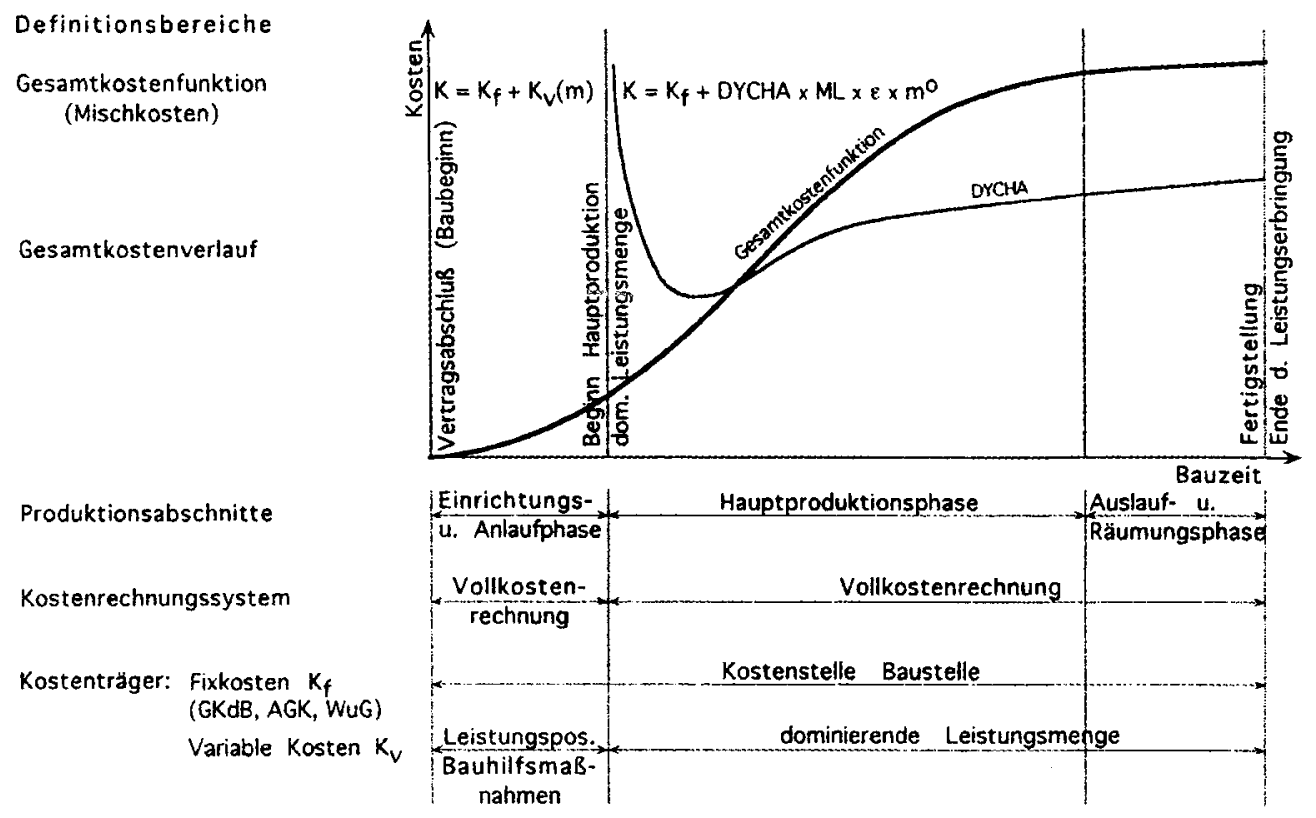

Abb. 3. Definitionsbereiche der Gesamtkostenfunktion

Die Neugliederung gegenüber den gegenwartsbezogenen Baustellen-Informationssystemen besteht darin, da $\beta$ es sich weder um ein bloßes Controllingsystem, noch um ein Modell mit Teilsystemen handelt, sondern um ein neues auf Ganzheitlichkeit und Transparenz ausgerichtetes und auf den modernen Methoden der Baubetriebs- und Wirtschaftswissenschaften aufbauendes baubetriebliches Informationssystem. Es gewährt zuverlässige Auskunft über aufklärende Informationen des aktuellen Bauprozesses und ermöglicht so rasche Entscheidungen.

\section{Literatur}

1. Bauer, H.: Baubetrieb 1: Einführung in die Rahmenbedingungen; Berlin/Heidelberg, SpringerVerl. 1992, S. 8 - 9.

2. Gehri, M.: Computerunterstützte Baustellenführung, Diss. ETH Zürich, vdf Verlag der Fachvereine an den schweizerischen Hochschulen und Techniken AG, Zürich, 1992, S. 42 - 45.

3. Bauer, H.: Baubetrieb 2: Bauablauf, Kosten, Störungen, Berlin/Heidelberg, Springer-Verl. 1992, S. $573-580$

4. Raeder, S.: Technologische und organisatorische Untersuchungen $\mathrm{zu}$ aktuellen Bauprozessen und akuten Baukosten - Eine prozeßorientierte homogene empirische Theorie des Baubetriebes; Diss. Vilnius Gediminas Technische Universität, Vilnius, 1997.

\section{STATYBOS IMONIU INFORMACINIUU SISTEMU NAUJOVIŠKAS SKIRSTYMAS PAGAL IMONĖS EKONOMINI IR PROCESU TECHNOLOGIJOS METODUS}

\section{S. Raeder}

\section{Santrauka}

Siandien statybos projekty igyvendinti nepanaudojus informacinès technikos yra neįmanoma. Š skirstyti $i$ keturis etapus: planavimą ir konstravimą, leidimy fazę, statybos paruošimą ir statybos vykdymą. Statybinés veiklos suskaidymas grubiai apima dvi pagrindines sritis techninę ir ekonominę.

Statybos i̇monès informacinès sistemos yra atviros dinaminès sistemos. Jose egzistuoja aktyvūs elementai, kurie yra susieti materialiais tiekimo dydžiais. Taip pat jos turi elementus, kurie nurodo santykị su aplinka, o taip pat ir su užsakovu. Sudarant šią sistemą reikia remtis tuo, kad statybos užsakymo ivykdymas vykty pagal sutartinius derinimus su tikslu gauti pelną. Visi nukrypimai nuo vadovavimo dydžiu turi būti kompensuojant pašalinti. Išvystyta statybos imonès informacinè sistema turi šiz reguliuojančią savybę. Tuomet ji apibūdinama kaip kibernetiné sistema.

Priešingai, i dabartị orientuotu statybos aikšteliu informacinių sistemų naujas suskaidymas yra toks, kad čia nekalbama nei apie gryną kontrolés sistemą, nei apie modelị su dalinemis sistemomis, bet apie statybos i̇monès informacinę sistemą, sukurtą pagal modernius statybos i̇monés ir ekonomikos moksly̨ metodus, nukreiptus sukurti pilnumą ir transparenciją. Tai garantuoja patikimą informaciją apie svarbu statybos procesą ir leidžia priimti skubius sprendimus. 


\section{INNOVATIVE DIVISION OF INFORMATION SYSTEMS OF BUILDING ENTERPRISES ACCORDING TO THE METHODS OF ECONOMY AND PROCESS TECHNOLOGY}

\section{S. Reader}

\section{Su m mary}

At the present time the implementation of building projects is impossible without using information technology. This process can be divided into 4 stages: 1) planning and constructing, 2) getting permissions, 3) building preparations, and 4) building peformance. Roughly speaking, decomposition of building activities includes two main spheres: technical and economic.

Building enterprise information systems are open dynamic systems. They consist of active elements connected by material supply quantities. They also include elements showing their relations to the environment and to the client. When arranging this system, one must have in mind that the implementation of a building order should follow contract agreements with the aim of getting profit. Therefore all the deviations from guiding quantities should be avoided by compensating them. A developed building enterprise information system implies this regulating property. In this case, it is referred as cybernetic system.
Contrary to the building site information systems oriented to the present time, the novelty of the new decomposition consists in that it does not deal with a pure control system and a model possessing partial systems, but with a building enterprise information system developed following modern methods of building enterprise as well as economic sciences and intended for achieving completeness and transparency. It ensures a reliable information pertaining to an important building process and permits to make timely decisions.

Siegfried READER. Doctor (Engr), Professor. Leipzig Higher School for Technology, Economy and Culture. Department of Building Organisation.

A graduate of Munich Technical University (1968). From 1973 to 1989 chief and project manager at large building sites. For 3 years he worked in strategic fields of business management. From 1992 onwards Professor of Leipzig Higher School for Technology, Economy and Culture. PhD at Vilnius Gediminas Technical University (1997). 